\title{
A switched adaptive observer for extended braking stiffness estimation*
}

\author{
Missie Aguado-Rojas ${ }^{1}$, William Pasillas-Lépine ${ }^{2}$ and Antonio Loría ${ }^{2}$
}

\begin{abstract}
We present a switched adaptive observer for the estimation of the tyre extended braking stiffness (XBS) during braking scenarios. The design of the observer is based on Burckhardt's friction model, and on a model of the wheel dynamics in which the XBS appears as one of the state variables. Asymptotic stability of the estimation error is established on the basis of dwell-time and persistency of excitation conditions. The approach is validated via numerical simulations considering realistic perturbations on the system's measured output.
\end{abstract}

\section{INTRODUCTION}

As vehicle safety-oriented control systems become more and more advanced, they also increase their dependence on accurate information about the state of the vehicle and its surroundings. For instance, the performance of advanced driver-assistance technologies such as the antilock braking system (ABS) is greatly influenced by the characteristics of the friction force between the tyre and the road. Therefore, by taking into account the external driving conditions of the vehicle, the effectiveness of such active safety systems can be greatly improved [1]. Tyre-road friction, however, cannot be directly measured in real-time, hence the estimation thereof has been an intensive research area in the last years. On the one hand, numerous different approaches for the estimation of the tyre-road friction coefficient and its optimal value, i.e., the value at which the maximum braking force can be obtained, have been proposed in the literature (see, e.g., [2], [3] and references therein). On the other hand, a different line of research focuses instead on the estimation of the so-called extended braking stiffness (XBS), which can be defined as the slope of the tyre-road friction coefficient with respect to the wheel slip at the operational point, and indicates the residual longitudinal friction force available to the driver. The interest of estimating the XBS is that, as opposed to the unknown optimal value of the tyre-road friction coefficient, the optimal value of the XBS is always equal to zero, hence an ABS control algorithm can be designed in order to maintain the XBS in a neighbourhood around zero.

The concept of maximizing the braking force in an ABS on the basis of the slope of the braking force with respect to the wheel slip was first introduced by Sugai et al. in [4], where XBS is identified by forcing a vibration via the brake actuator and analyzing the frequency characteristics

\footnotetext{
*This article is supported by the Dept. STIC of Univ. Paris-Saclay, France. The work of the first author is supported by CONACYT and SEP, Mexico.

${ }^{1} \mathrm{M}$. Aguado-Rojas is with Université Paris-Sud, Université Paris-Saclay, L2S-CentraleSupélec, 91192 Gif-sur-Yvette, France.

missie.aguadoel2s.centralesupelec.fr

${ }^{2}$ W. Pasillas-Lépine and A. Loría are with CNRS, L2S-CentraleSupélec, 91192 Gif-sur-Yvette, France.

\{pasillas\}\{loria\}@l2s.centralesupelec.fr
}

of a resonance system composed of the vehicle body, the wheel and the road surface. The term extended braking stiffness was later coined by Umeno in [5], where the XBS is estimated via the instrumental variable method based on the frequency characteristics of a tyre vibration model linearized around a constant-velocity operating point. In [6], the XBS is (implicitly) assumed to be a constant parameter and estimated by applying the recursive least squares algorithm to wheel rotational velocities. In [7], in order to estimate the maximum friction coefficient, the concept of XBS is used to signal the entrance of the tyre into a different road surface and to distinguish one type of road from another. The XBS is estimated using elementary diagnostic tools and algebraic methods for filtering and estimating derivatives of noisy signals (whose main difficulty is to achieve a good tradeoff between filtering and reactivity), and the estimation results are accurate only within a certain validity range. In [8], a model of the wheel acceleration dynamics in which the XBS appears as one of the state variables was introduced. In order to cope with uncertainty in road conditions, the unknown parameters of the model are assumed to be constant and regarded as state variables with dynamics equal to zero. The XBS is estimated using an asymptotic state observer for the augmented system. In that work, however, knowledge of upper and lower bounds of the unknown parameters is still required, hence the observer might not be robust to large changes in road conditions. In this paper, we exploit the model introduced in [8] and present an adaptive switched observer for the estimation of the XBS. The proposed approach does not require any a priori knowledge of the parameters related to the road conditions, and has an overall better performance than the one previously proposed in [8].

\section{Single-WHEEL SYSTEM MODELLING}

In this paper, we employ a basic single-wheel model and consider the forces acting on the longitudinal direction only. This model, despite its simplicity, is known to provide a simple yet sufficiently rich description of the braking dynamics, hence it is widely encountered in the literature of active braking control systems (see, e.g., [9, Ch. 2]).

\section{A. Wheel dynamics}

The dynamics of the angular velocity $\omega$ of the wheel is described by

$$
I \dot{\omega}=-R F_{x}+T,
$$

where $I$ is the inertia of the wheel, $R$ is its effective rolling radius, $F_{x}$ denotes the longitudinal tyre force, and $T=T_{e}-T_{b}$ is the torque applied to the wheel, composed by the engine torque $T_{e}$ and the brake torque $T_{b}$. In the 


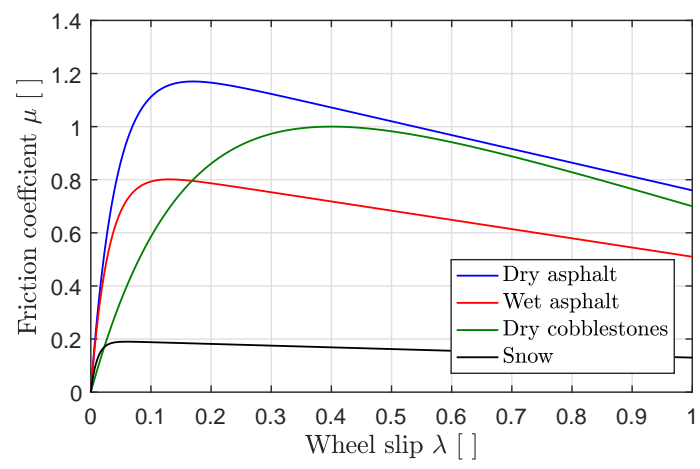

Fig. 1. Typical tyre-road friction curves obtained using Burckhardt's model for different road surface conditions. The corresponding parameter values are given in Table I.

following, it is assumed that the clutch is open during an ABS braking manoeuver, hence the engine torque can be neglected. The brake torque is given by $T_{b}=\gamma_{b} P_{b}$, where $\gamma_{b}$ denotes the brake efficiency, and $P_{b}$ the brake pressure.

The longitudinal tyre force $F_{x}$ is modelled as

$$
F_{x}=\mu(\lambda) F_{z},
$$

where $F_{z}$ is the vertical force at the tyre-road contact point and $\mu(\lambda)$ denotes the tyre-road friction (or adhesion) coefficient, i.e., it describes the tyre capability of transferring the vertical load to the ground in the longitudinal direction. The friction coefficient depends nonlinearly on the longitudinal wheel slip $\lambda$ defined as

$$
\lambda=\frac{R \omega-v_{x}}{v_{x}},
$$

where $v_{x}>0$ is the longitudinal speed of the vehicle, and $R \omega$ is the linear speed of the tyre at the wheel-ground contact point. In other words, $\lambda$ denotes the normalized relative velocity between the road and the tyre. A zero slip corresponds to a pure rolling wheel, while the presence of a non-zero slip is due, in general, to traction and braking forces exerted on the tyre. In particular, the region $\lambda<0$ corresponds to braking, and $\lambda=-1$ to a locked wheel.

The typical behaviour of the friction coefficient curve $\mu(\lambda)$ for different road conditions is illustrated in Fig. 1. It is a smooth function symmetric about the origin (only the region $\lambda>0$ that corresponds to traction is shown) that satisfies $\mu(0)=0, \frac{d \mu(0)}{d \lambda}>0$, and has a single maximum at $\lambda=\lambda^{*}$. Note that the optimal wheel slip $\lambda^{*}$, i.e., the value for which the maximum longitudinal tyre force is transferred to the ground, changes depending on the road conditions.

Several models of the tyre-road friction coefficient are available in the literature (see, e.g., [10] and references therein). In this work, the tyre characteristic model proposed by Burckhardt [11] is employed, i.e., the friction coefficient is modelled as

$$
\mu(\lambda)=c_{1}\left(1-\exp \left(-c_{2} \lambda\right)\right)-c_{3} \lambda,
$$

where the coefficients $c_{i}$ are "constants" that depend on the road conditions, on the tyre characteristics, and on the vehicle operational conditions [10]. Therefore, by changing the
TABLE I

BURCKHARDT'S MODEL PARAMETERS [12, P. 322]

\begin{tabular}{cccc}
\hline Road condition & $c_{1}$ & $c_{2}$ & $c_{3}$ \\
\hline Dry asphalt & 1.2801 & 23.99 & 0.52 \\
Wet asphalt & 0.857 & 33.822 & 0.347 \\
Cobblestone & 1.3713 & 6.4565 & 0.6691 \\
Snow & 0.1946 & 94.129 & 0.0646 \\
\hline
\end{tabular}

values of these three parameters, many different conditions can be modelled.

\section{B. Simplified wheel slip and wheel acceleration dynamics}

Define as state variables the wheel slip $x_{1}:=\lambda$ and the wheel acceleration offset

$$
x_{2}:=R \dot{\omega}-a_{x}(t),
$$

i.e., the difference between the longitudinal acceleration of the vehicle $a_{x}(t)=\dot{v}_{x}(t)$ and the linear acceleration of the tyre $R \dot{\omega}$ at the wheel-ground contact point. The dynamics of the system is given by

$$
\begin{aligned}
& \dot{x}_{1}=\frac{1}{v_{x}(t)}\left(-a_{x}(t) x_{1}+x_{2}\right) \\
& \dot{x}_{2}=-\frac{R^{2}}{I} F_{z} \frac{\mu^{\prime}\left(x_{1}\right)}{v_{x}(t)}\left(-a_{x}(t) x_{1}+x_{2}\right)-\frac{R}{I} \gamma_{b} \dot{P}_{b}-\dot{a}_{x}(t),
\end{aligned}
$$

where

$$
\mu^{\prime}(\lambda)=\frac{d \mu(\lambda)}{d \lambda}
$$

is the tyre extended braking stiffness (XBS), which indicates the residual longitudinal friction force available to the driver. Note that, as opposed to the maximum friction coefficient $\mu\left(\lambda^{*}\right)$, which depends on the road conditions and is usually unknown, the XBS that corresponds to the optimal wheel slip is always known, i.e., $\mu^{\prime}\left(\lambda^{*}\right)=0$.

During an ABS braking manoeuver, the longitudinal acceleration of the vehicle remains almost constant and close to the maximal value $a_{x}^{*}$ allowed by the road conditions, and the wheel slip remains relatively small. Under these conditions, the approximations $\dot{a}_{x}(t) \simeq 0$ and $\left(-a_{x}(t) x_{1}+x_{2}\right) \simeq x_{2}$ are sensible. While the equalities are true only when $v_{x}(t)$ is constant, the approximations remain reasonable during the activation of the ABS. This leads to the simplified dynamics

$$
\begin{aligned}
\dot{x}_{1} & =\frac{1}{v_{x}(t)} x_{2} \\
\dot{x}_{2} & =-\frac{a}{v_{x}(t)} x_{2} \mu^{\prime}\left(x_{1}\right)-b u,
\end{aligned}
$$

where $a=\left(R^{2} / I\right) F_{z}, b=(R / I) \gamma_{b}$, and $u=\dot{P}_{b}$. The longitudinal speed of the vehicle $v_{x}(t)$ is assumed to be strictly positive and considered as an external variable of the model. 


\section{XBS dynamics}

Given a suitable choice for the tyre-road friction model, system (3) can be used to develop a simple model wherein the tyre XBS appears directly as a state variable. Such a model can then be used to design a state observer in order to estimate the variable of interest.

To that end, let the wheel acceleration offset $z_{1}:=x_{2}$ and the XBS $z_{2}:=\mu^{\prime}\left(x_{1}\right)$ be defined as new state variables. From (1), recalling that $x_{1}=\lambda$, one has

$$
\begin{aligned}
& \mu^{\prime}\left(x_{1}\right)=c_{1} c_{2} \exp \left(-c_{2} x_{1}\right)-c_{3} \\
& \mu^{\prime \prime}\left(x_{1}\right)=-c_{2} c_{1} c_{2} \exp \left(-c_{2} x_{1}\right)
\end{aligned}
$$

from which the following relation can be established

$$
\mu^{\prime \prime}\left(x_{1}\right)=-c_{2} \mu^{\prime}\left(x_{1}\right)-c_{2} c_{3} .
$$

The above relation, along with system (3), leads to the dynamics

$$
\begin{aligned}
& \dot{z}_{1}=-\frac{a}{v_{x}(t)} z_{1} z_{2}-b u \\
& \dot{z}_{2}=\left(c z_{2}+d\right) \frac{1}{v_{x}(t)} z_{1},
\end{aligned}
$$

where $c=-c_{2}, d=-c_{2} c_{3}$.

The objective of this paper is to design an observer to estimate the (unmeasurable) XBS $z_{2}$ under unknown road conditions, i.e., unknown $c_{2}$ and $c_{3}$, using the available information of the (measurable) wheel acceleration offset $z_{1}$. The longitudinal speed of the vehicle $v_{x}(t)$, and the constant parameters $a, b$ are assumed to be known as well.

\section{ADAPTIVE OBSERVER DESIGN}

As a first step towards the observer design, consider the linear change of coordinates

$$
\begin{aligned}
& w_{1}=z_{1} \\
& w_{2}=z_{2}+\frac{c}{a} z_{1}
\end{aligned}
$$

that transforms the system (4) into

$$
\begin{aligned}
& \dot{w}_{1}=\frac{w_{1}}{v_{x}(t)}\left(c w_{1}-a w_{2}\right)-b u \\
& \dot{w}_{2}=-\frac{b c}{a} u+\frac{w_{1}}{v_{x}(t)} d,
\end{aligned}
$$

which is bilinear. We may thus rely on observer design methods for systems that are linear in the unmeasured variables - see e.g., [13], as well as [14]-[16]. To better see this, let the above equations be rewritten in the form

$$
\begin{aligned}
\dot{w} & =A(t, y) w+B u+\Psi(t, u, y) \theta \\
y & =C w
\end{aligned}
$$

with $w=\left(\begin{array}{ll}w_{1} & w_{2}\end{array}\right)^{\top}$,

$$
A(t, y)=\frac{w_{1}}{v_{x}(t)}\left(\begin{array}{cc}
0 & -a \\
0 & 0
\end{array}\right):=\frac{w_{1}}{v_{x}(t)} A^{\prime}, \quad B=\left(\begin{array}{c}
-b \\
0
\end{array}\right) \text {, }
$$

$$
\Psi(t, u, y)=\left(\begin{array}{cc}
\frac{w_{1}^{2}}{v_{x}(t)} & 0 \\
-\frac{b}{a} u & \frac{w_{1}}{v_{x}(t)}
\end{array}\right), \quad C=\left(\begin{array}{ll}
1 & 0
\end{array}\right),
$$

and $\theta=\left(\begin{array}{ll}c & d\end{array}\right)^{\top}$. We assume that the measured output is $y=w_{1}=x_{2}$, i.e., the wheel acceleration offset (2). Then, following [14], we introduce the switched adaptive observer

$$
\begin{aligned}
\dot{\hat{w}}= & A(t, y) \hat{w}+B u+\Psi(t, u, y) \hat{\theta} \\
& +\left(K(t, y)+\Upsilon \Gamma \Upsilon^{\top} C^{\top}\right)(y-C \hat{w}) \\
\dot{\hat{\theta}}= & \Gamma \Upsilon^{\top} C^{\top}(y-C \hat{w}) \\
\dot{\Upsilon}= & (A(t, y)-K(t, y) C) \Upsilon+\Psi(t, u, y)
\end{aligned}
$$

with $\Gamma=\Gamma^{\top}>0$ and

$$
K(t, y):=\frac{w_{1}}{v_{x}(t)} K^{\prime}\left(w_{1}\right),
$$

where

$$
K^{\prime}\left(w_{1}\right)= \begin{cases}\left(\begin{array}{l}
k_{1}^{+} \\
k_{2}^{+}
\end{array}\right), & \text {if } w_{1}>0 \\
\left(\begin{array}{l}
k_{1}^{-} \\
k_{2}^{-}
\end{array}\right), & \text {if } w_{1}<0\end{cases}
$$

and $k_{1,2}^{ \pm}$are design parameters. The states of the original system are estimated via the inverse change of coordinates

$$
\begin{aligned}
& \hat{z}_{1}=\hat{w}_{1} \\
& \hat{z}_{2}=\hat{w}_{2}-\frac{\hat{c}}{a} \hat{w}_{1},
\end{aligned}
$$

which depends on the value of the estimated parameter $\hat{c}$. It is clear that if $\hat{w} \rightarrow w$ and $\hat{\theta} \rightarrow \theta$, then $\hat{z} \rightarrow z$.

In order to discuss the stability properties of the observer we make use of the following lemma.

Lemma 1: Consider system (5) and the switching function (7). If the gains $k_{1,2}^{ \pm}$in (8) satisfy

$$
k_{1}^{+}>0, \quad k_{2}^{+}<0, \quad k_{1}^{-}=-k_{1}^{+}<0, \quad k_{2}^{-}=k_{2}^{+}<0,
$$

then the origin of the system

$$
\dot{\eta}=(A(t, y)-K(t, y) C) \eta
$$

is stable. Moreover, if the switching signal $\sigma\left(w_{1}\right)$ that selects the observer gains admits a strictly positive minimal dwell time, i.e., if any two consecutive switchings are separated by no less than $\tau_{D}>0$, then, for the system (9), the origin is globally asymptotically stable.

Proof: The proof is inspired by Hoàng et al. [8]. From (5), (7) and (9) we have

$$
\dot{\eta}=\frac{w_{1}}{v_{x}(t)}\left(A^{\prime}-K^{\prime}\left(w_{1}\right) C\right) \eta .
$$

Note that if the right-hand side of the above equation is multiplied by $v_{x}(t) / w_{1}$, one obtains a linear time-varying system. Defining the new time-scale

$$
\tau(t):=\int_{0}^{t} \frac{\left|w_{1}(\varsigma)\right|}{v_{x}(\varsigma)} d \varsigma
$$


ensures that $d t / d \tau>0$, independently of the value of $w_{1}$. Now, since for any function $\varphi: \mathbb{R} \rightarrow \mathbb{R}^{n}$ one has

$$
\frac{d \varphi}{d \tau}=\frac{d \varphi}{d t} \frac{d t}{d \tau}=\frac{d \varphi}{d t} \frac{v_{x}(t)}{\left|w_{1}(t)\right|}
$$

the dynamics of $\eta$ in the new time-scale $\tau$ is given by

$$
\begin{aligned}
\frac{d \eta}{d \tau} & =\frac{w_{1}}{\left|w_{1}\right|}\left(A^{\prime}-K^{\prime}\left(w_{1}\right) C\right) \eta(\tau) \\
& =\operatorname{sgn}\left(w_{1}\right)\left(A^{\prime}-K^{\prime}\left(w_{1}\right) C\right) \eta(\tau)
\end{aligned}
$$

hence we obtain the switched linear dynamics

$$
\frac{d \eta}{d \tau}=A_{\sigma\left(w_{1}\right)} \eta(\tau)
$$

where

$$
A_{\sigma\left(w_{1}\right)}= \begin{cases}A_{+}=\left(\begin{array}{cc}
-k_{1}^{+} & -a \\
-k_{2}^{+} & 0
\end{array}\right), & \text { if } w_{1}>0 \\
A_{-}=\left(\begin{array}{ll}
k_{1}^{-} & a \\
k_{2}^{-} & 0
\end{array}\right), & \text { if } w_{1}<0 .\end{cases}
$$

The conditions $k_{1}^{+}>0, k_{2}^{+}<0, k_{1}^{-}<0, k_{2}^{-}<0$ ensure that the matrices $A_{+}, A_{-}$are Hurwitz, and a direct computation shows that the pairs $\left(A_{+}, C\right),\left(A_{-}, C\right)$ are observable. Hence, we know that there exist two symmetric positivedefinite matrices $P_{+}, P_{-}$such that $A_{+}^{\top} P_{+}+P_{+} A_{+}=-C^{\top} C$ and $A_{-}^{\top} P_{-}+P_{-} A_{+}=-C^{\top} C$ are satisfied. Furthermore, by imposing $k_{1}^{-}=-k_{1}^{+}, k_{2}^{-}=k_{2}^{+}$the above Lyapunov equations admit a common solution, that is $P_{+}=P_{-}=: P$ defines a nonstrict (because $C^{\top} C$ is positive semidefinite) common Lyapunov function for the switched system (10). Under these conditions, we can invoke [17, Cor. 1] to conclude stability of (10) and, consequently, stability of (9). Moreover, in view of the assumption that the switching signal $\sigma\left(w_{1}(t)\right)$ admits a strictly positive minimal dwell time, it follows from [17, Th. 4] that for the system (10) the origin is exponentially stable, uniformly with respect to the switching signal. Thus, for the system (9), i.e., in the original timescale, the origin is globally asymptotically stable.

The stability properties of the observer are summarized in the following theorem.

Theorem 1: Consider system (5) and observer (6). Let $\tilde{w}:=\hat{w}-w$ and $\tilde{\theta}:=\hat{\theta}-\theta$. Let the observer gains $k_{1,2}^{ \pm}$ in (7) satisfy

$$
k_{1}^{+}>0, \quad k_{2}^{+}<0, \quad k_{1}^{-}=-k_{1}^{+}<0, \quad k_{2}^{-}=k_{2}^{+}<0 .
$$

Assume that the switching signal $\sigma\left(w_{1}\right)$ that selects the observer gains admits a strictly positive minimal dwell time. If $\Psi(t, u(t), y(t))$ is persistently exciting, then, for the closed-loop system, the origin $(\tilde{w}, \tilde{\theta})=(0,0)$ is globally asymptotically stable.

Proof: The proof is inspired by Zhang [14]. After (5), (6a) and (6b), the dynamics of the observation error is

$$
\dot{\tilde{w}}=(A(t, y)-K(t, y) C) \tilde{w}+\Psi(t, u, y) \tilde{\theta}+\Upsilon \dot{\tilde{\theta}} .
$$

Let $\eta=\tilde{w}-\Upsilon \tilde{\theta}$. From the above equation and (6c) we have

$$
\begin{aligned}
\dot{\eta} & =(A(t, y)-K(t, y) C) \tilde{w}+\Psi(t, u, y) \tilde{\theta}-\dot{\Upsilon} \tilde{\theta} \\
& =(A(t, y)-K(t, y) C) \eta .
\end{aligned}
$$

From Lemma 1, for this system the origin is globally asymptotically stable. Now, from (6b) and the definition of $\eta$, the dynamics of the parameter estimation error is

$$
\dot{\tilde{\theta}}=-\Gamma \Upsilon^{\top} C^{\top} C(\Upsilon \tilde{\theta}+\eta) \text {. }
$$

Consider the homogeneous part of (12), that is

$$
\dot{\tilde{\theta}}=-\Gamma \Upsilon^{\top} C^{\top} C \Upsilon \tilde{\theta} .
$$

If $\Psi(t, u(t), y(t))$ is persistently exciting (PE), i.e., if there exist positive constants $\mu_{0}, T_{0}$ such that for all $t$

$$
\int_{t}^{t+T_{0}} \Psi(\varsigma, u(\varsigma), y(\varsigma))^{\top} \Psi(\varsigma, u(\varsigma), y(\varsigma)) d \varsigma \geq \mu_{0} \mathbf{I},
$$

then $\Upsilon(t, u(t), y(t))$ generated by (6c) is also PE [18]. It follows that the origin of system (13) is globally exponentially stable and system (12) is input-to-state stable with respect to the input $\eta$. Global asymptotic stability of $(\eta, \tilde{\theta})=(0,0)$ follows. Finally, to see that the same property holds for $(\tilde{w}, \tilde{\theta})=(0,0)$ we observe that $\tilde{w}=\eta+\Upsilon \tilde{\theta}$.

\section{Simulation RESUlts}

The performance of the proposed observer has been tested via numerical simulations considering the (nonsimplified) single-wheel model described in Section II-A. A braking scenario of a vehicle traveling with an initial speed of $25 \mathrm{~m} / \mathrm{s}$ and a constant deceleration of $1.96 \mathrm{~m} / \mathrm{s}^{2}$ is illustrated in Figs. 2 to 9 . Changes in road conditions occur at times $t=3$ and $t=6$ : during the first part of the simulation the wheel runs on dry asphalt, then on wet asphalt, and finally on dry concrete. For the purpose of generating meaningful signals, the system is driven via a five-phase hybrid ABS control algorithm (see [19], [20] for a full description) that modulates the brake pressure based on measurements of the wheel acceleration offset. Persistency of excitation of $\Psi(t, u(t), y(t))$ under such scenario is verified in Fig. 2, which shows the eigenvalues of the left-hand side of (14) for $T_{0}=1$. Recall that $\Psi(\cdot)$ is PE if and only if

$$
\varrho_{\min }\left\{\int_{t}^{t+T_{0}} \Psi(\cdot)^{\top} \Psi(\cdot) d \varsigma\right\} \geq \mu_{0}>0
$$

for all $t$, where $\varrho_{\min }\{\cdot\}$ denotes the minimal eigenvalue of the argument. The observer was tested under ideal conditions, i.e., without measurement noise or other external disturbances, as well as under a more realistic scenario considering a velocity-dependent perturbation in the system's measured output (Fig. 3). That is, the measurement of the wheel acceleration offset is perturbed by periodic (in one mechanical revolution) oscillations caused by imperfections in the wheel-speed sensors used in automotive applications (see [21] and references therein).

The estimation results obtained under the ideal scenario are illustrated in Figs. 4 to 6 . A quick convergence to zero of both the state and parameter estimation errors $\tilde{w}$, $\tilde{\theta}$ can be observed (Fig. 4), even after the abrupt transitions between different types of roads. Consequently, the estimated XBS also converges to its true value. In Figs. 5 and 6 , we show a comparison between the XBS estimated via 


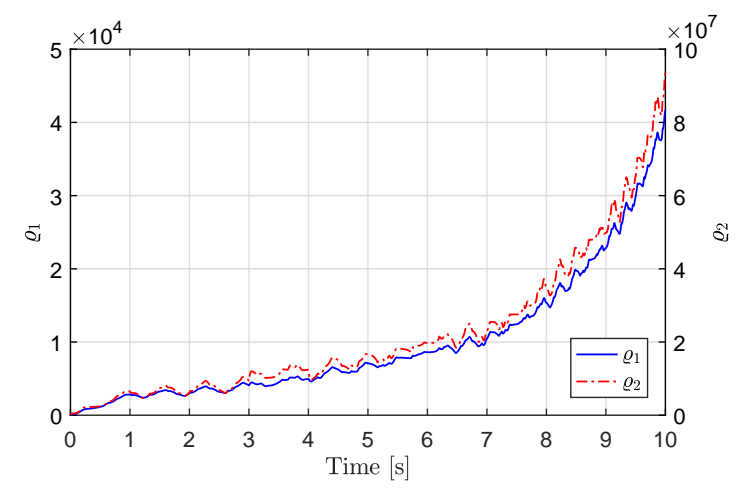

Fig. 2. Eigenvalues of $\int_{t}^{t+T_{0}} \Psi(\varsigma, u(\varsigma), y(\varsigma))^{\top} \Psi(\varsigma, u(\varsigma), y(\varsigma)) d \varsigma$.

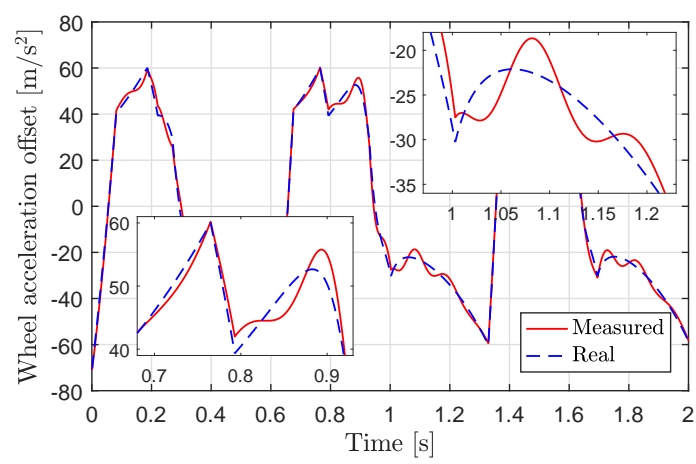

Fig. 3. Wheel acceleration offset: real signal vs perturbed measurement.

the adaptive observer presented in this paper and the one estimated via the augmented-state observer (ASO) proposed in [8]. The adaptive observer exhibits better robustness properties to changes in road conditions than the ASO, and a better performance both during transient and steady-state conditions. Figs. 7 to 9 illustrate the results obtained under the perturbed scenario. As could be expected, the estimation errors $\tilde{w}, \tilde{\theta}$ are perturbed due to the error in the system's measured output, but they approach zero as the perturbation vanishes (because the perturbation is velocity-dependent, its amplitude decreases as the vehicle velocity is reduced). Therefore, with the adaptive observer the estimated XBS closely approaches its true value. Such is not the case for the ASO, which displays greater sensitivity to the error in the system's measured output, and whose estimation error does not converge to zero even as the perturbation vanishes. The results thus validate the effectiveness of the proposed approach.

\section{CONCLUSION}

This paper presented a switched adaptive observer for the estimation of the tyre extended braking stiffness. The design of the observer is based on a simplified dynamic model that retains a good degree of accuracy during an ABScontrolled braking scenario. The performance of the observer was tested via numerical simulations with satisfactory results under ideal and perturbed conditions. Future work will focus on the experimental evaluation of the proposed approach.

\section{REFERENCES}

[1] C. Ahn, H. Peng, and H. E. Tseng, "Robust estimation of road frictional coefficient," IEEE Transactions on Control Systems Technology, vol. 21, no. 1, pp. 1-13, Jan 2013.

[2] S. Müller, M. Uchanski, and K. Hedrick, "Estimation of the maximum tire-road friction coefficient," Journal of Dynamic Systems, Measurement, and Control, vol. 125, no. 4, pp. 607-617, Dec 2003.

[3] R. Rajamani, N. Piyabongkarn, J. Lew, K. Yi, and G. Phanomchoeng, "Tire-road friction-coefficient estimation," IEEE Control Systems, vol. 30, no. 4, pp. 54-69, Aug 2010.

[4] M. Sugai, H. Yamaguchi, M. Miyashita, T. Umeno, and K. Asano, "New control technique for maximizing braking force on antilock braking system," Vehicle System Dynamics, vol. 32, no. 4-5, pp. 299 312, 1999.

[5] T. Umeno, "Estimation of tire-road friction by tire rotational vibration model," R\&D Review of Toyota CRDL, vol. 37, no. 3, 2002.

[6] E. Ono, K. Asano, M. Sugai, S. Ito, M. Yamamoto, M. Sawada, and Y. Yasui, "Estimation of automotive tire force characteristics using wheel velocity," Control Engineering Practice, vol. 11, no. 12, pp. 1361-1370, 2003.

[7] J. Villagra, B. d'Andréa Novel, M. Fliess, and H. Mounier, "A diagnosis-based approach for tire-road forces and maximum friction estimation," Control Engineering Practice, vol. 19, no. 2, pp. $174-$ $184,2011$.

[8] T. B. Hoàng, W. Pasillas-Lépine, A. De Bernardinis, and M. Netto, "Extended braking stiffness estimation based on a switched observer, with an application to wheel-acceleration control," IEEE Transactions on Control Systems Technology, vol. 22, no. 6, pp. 2384-2392, Nov 2014.

[9] S. M. Savaresi and M. Tanelli, Active braking control systems design for vehicles, ser. Advances in Industrial Control. Springer-Verlag London, 2010.

[10] C. Canudas de Wit, P. Tsiotras, E. Velenis, M. Basset, and G. Gissinger, "Dynamic friction models for road/tire longitudinal interaction," Vehicle System Dynamics, vol. 39, no. 3, pp. 189-226, 2003

[11] M. Burckhardt, Fahrwerktechnik: Radschlupf-Regelsysteme. VogelVerlag, 1993.

[12] U. Kiencke and L. Nielsen, Automotive control systems: For engine, driveline, and vehicle, 2nd ed. Springer-Verlag Berlin Heidelberg, 2005.

[13] G. Besançon, "A viewpoint on observability and observer design for nonlinear systems," in New Directions in Nonlinear Observer Design, ser. Lecture Notes in Control and Information Sciences, H. Nijmeijer and T. Fossen, Eds. Springer-Verlag London, 1999, vol. 244, pp 3-22.

[14] Q. Zhang, "Adaptive observer for multiple-input-multiple-output (MIMO) linear time-varying systems," IEEE Transactions on Automatic Control, vol. 47, no. 3, pp. 525-529, Mar 2002.

[15] — "An adaptive observer for sensor fault estimation in linear time varying systems," IFAC Proceedings Volumes, vol. 38, no. 1, pp. 137142, 2005, 16th IFAC World Congress.

[16] X. Li, Q. Zhang, and H. Su, "An adaptive observer for joint estimation of states and parameters in both state and output equations," International Journal of Adaptive Control and Signal Processing, vol. 25, no. 9, pp. 831-842, 2011.

[17] J. P. Hespanha, "Uniform stability of switched linear systems: extensions of lasalle's invariance principle," IEEE Transactions on Automatic Control, vol. 49, no. 4, pp. 470-482, April 2004.

[18] E. Panteley, A. Loría, and A. Teel, "Relaxed persistency of excitation for uniform asymptotic stability," IEEE Transactions on Automatic Control, vol. 46, no. 12, pp. 1874-1886, Dec 2001.

[19] W. Pasillas-Lépine, "Hybrid modeling and limit cycle analysis for a class of five-phase anti-lock brake algorithms," Vehicle System Dynamics, vol. 44, no. 2, pp. 173-188, 2006.

[20] M. Gerard, W. Pasillas-Lépine, E. de Vries, and M. Verhaegen, "Improvements to a five-phase ABS algorithm for experimental validation," Vehicle System Dynamics, vol. 50, no. 10, pp. 1585-1611, 2012

[21] M. Aguado-Rojas, W. Pasillas-Lépine, A. Loría, and A. De Bernardinis, "Angular velocity estimation from incremental encoder measurements in the presence of sensor imperfections," in Proceedings of the 20th IFAC World Congress, July 9-14 2017, pp. 6153-6158. 

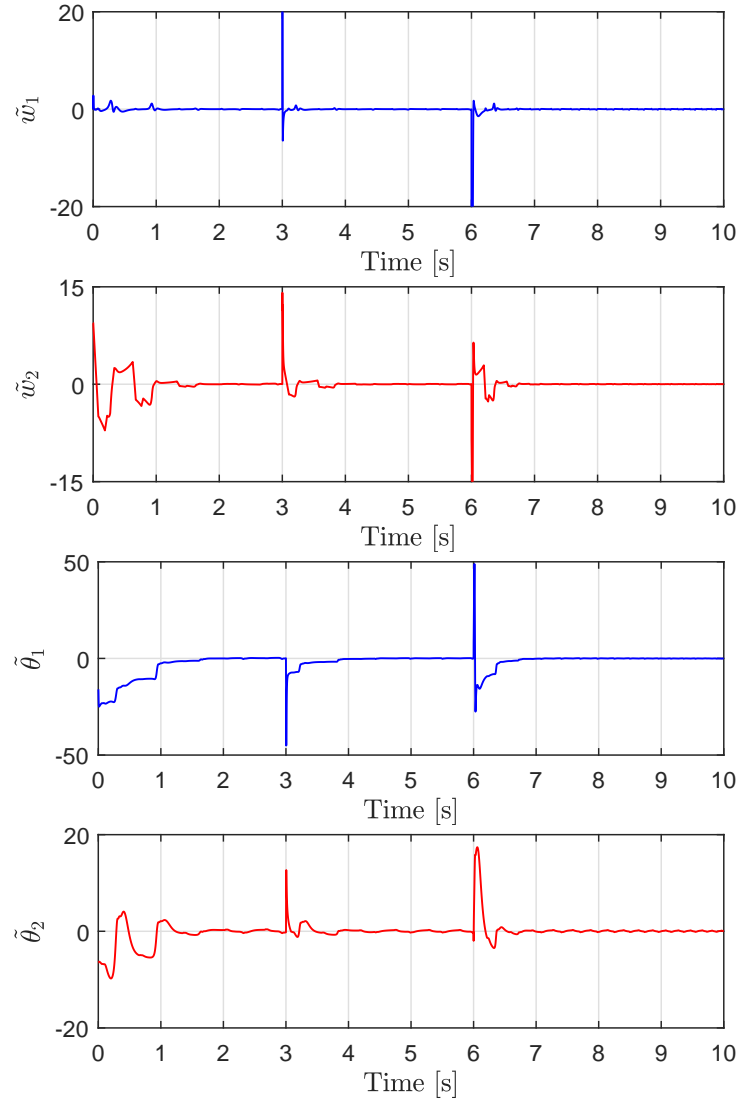

Fig. 4. Ideal scenario: state and parameter estimation errors.

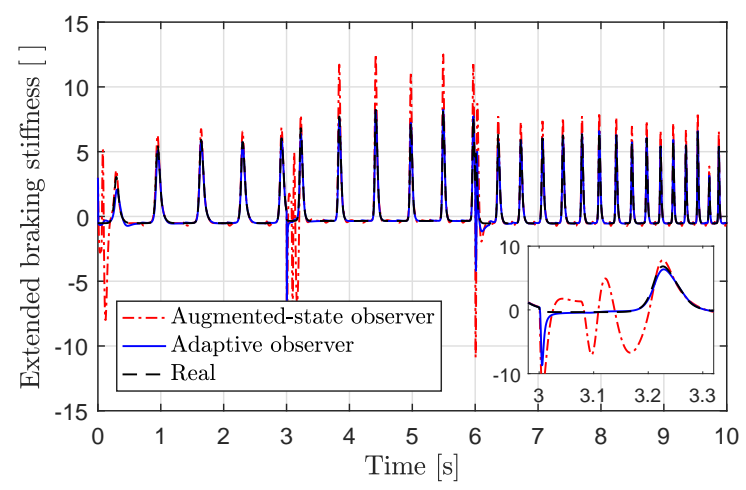

Fig. 5. Ideal scenario: real vs estimated XBS.

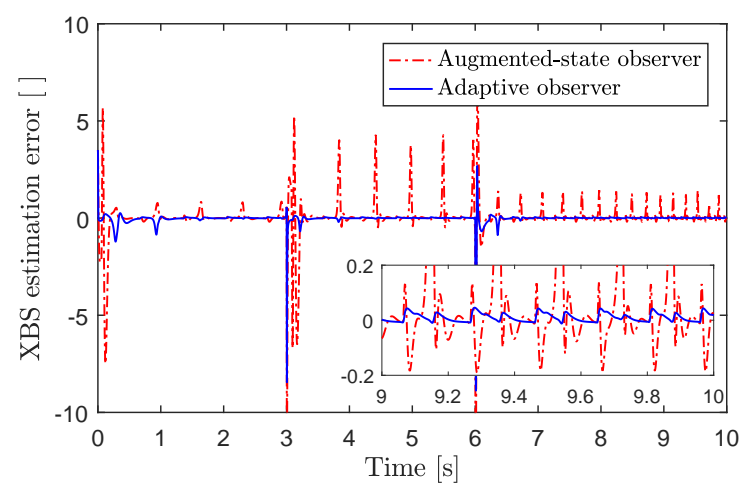

Fig. 6. Ideal scenario: XBS estimation error
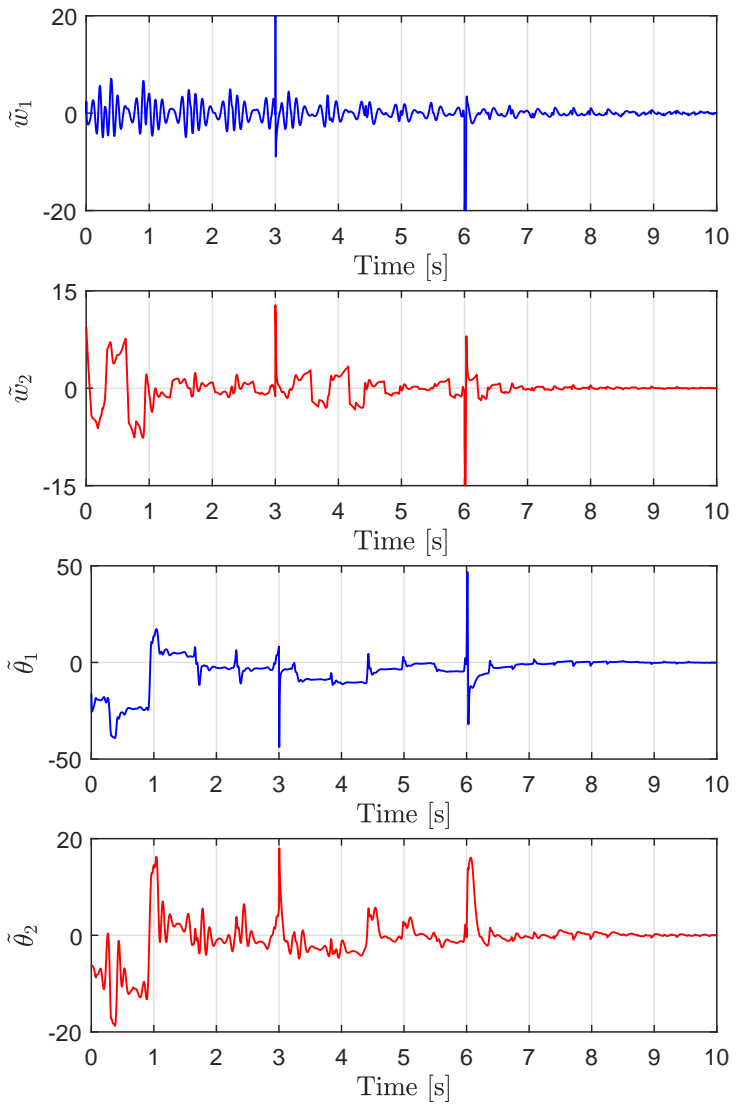

Fig. 7. Perturbed scenario: state and parameter estimation errors.

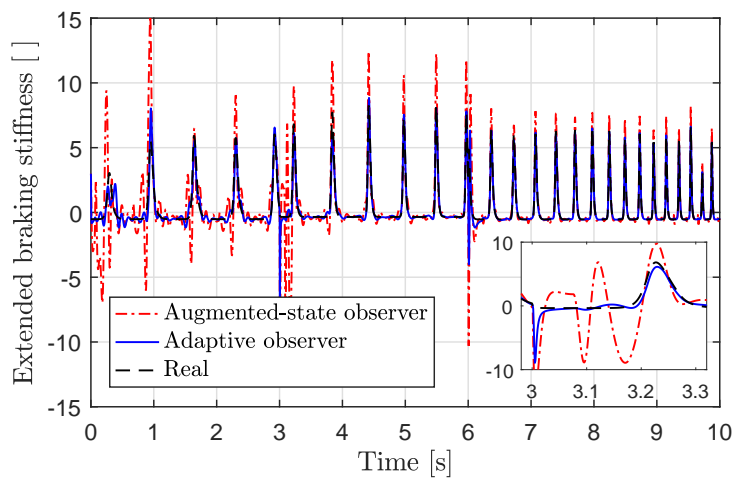

Fig. 8. Perturbed scenario: real vs estimated XBS.

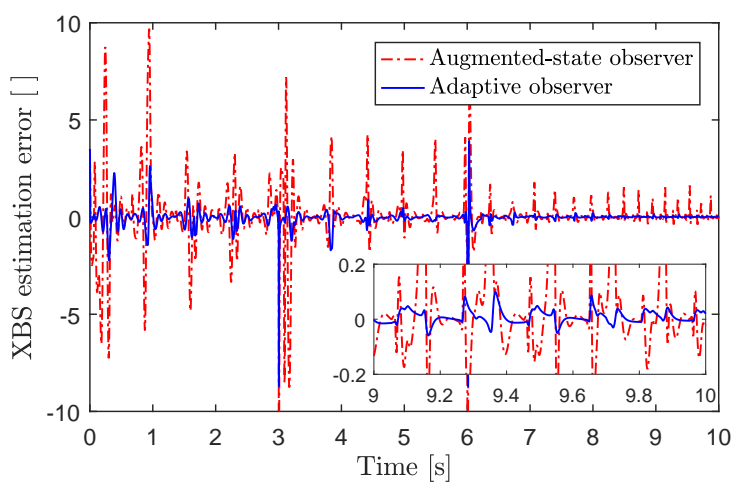

Fig. 9. Perturbed scenario: XBS estimation error. 